\section{$\underset{\substack{\text { hommes } \\ \text { \& migrations }}}{ }$}

\section{Hommes \& migrations}

Revue française de référence sur les dynamiques

migratoires

1307 | 2014

L'Afrique qualifiée dans la mondialisation

\title{
Enseigner les migrations en géographie : un état des lieux
}

\section{Tangui Pennec}

\section{OpenEdition \\ Journals}

Édition électronique

URL : http://journals.openedition.org/hommesmigrations/2915

DOI : 10.4000/hommesmigrations.2915

ISSN : 2262-3353

Éditeur

Musée national de l'histoire de l'immigration

Édition imprimée

Date de publication : 1 juillet 2014

Pagination : 170-175

ISBN : 978-2-919040-28-5

ISSN : $1142-852 X$

\section{Référence électronique}

Tangui Pennec, "Enseigner les migrations en géographie : un état des lieux », Hommes \& migrations [En ligne], 1307 | 2014, mis en ligne le 15 janvier 2015, consulté le 22 septembre 2020. URL : http:// journals.openedition.org/hommesmigrations/2915; DOI : https://doi.org/10.4000/ hommesmigrations.2915 


\section{ENSEIGNER LES MIGRATIONS EN GÉOGRAPHIE UN ÉTAT DES LIEUX'}

TANGUI PENNEC, agrégé de géographie, doctorant en géographie-géopolitique (IFG, Paris-VIII), professeur d'histoire-géographie au lycée Jules-Verne, Cergy-le-Haut (95).

$\mathrm{L}^{2}$ a recherche a permis à la géographie de se doter Lde nouveaux outils, non seulement pour penser globalement les déplacements des hommes, mais aussi pour comprendre les nouvelles territorialités à l'œuvre dans un monde de plus en plus réticulaire. Mais, si les migrations sont aujourd'hui au centre des réflexions géographiques, elles ont longtemps été une forme d'impensé dans la géographie traditionnelle française, dont le corpus théorique, façonné par son fondateur Paul Vidal de la Blache (1845-1918), était focalisé sur la fixité spatiale (l'attachement à la terre) et le temps long. Une "géographie des migrations" émerge certes plus tard sous la plume de Pierre Georges², mais c'est dans les années 1980 que s'opère un véritable "tournant géographique" dans l'étude des migrations, alors même que le processus de mondialisation s'accélère. À la faveur des travaux de Gildas Simon ${ }^{3}$ et grâce à l'apport d'autres disciplines (anthropologie, sociologie avec Alain Tarrius) notamment dans le cadre du laboratoire $\mathrm{Mi}$ grinter, l'analyse des migrations prend davantage en compte la complexité du rapport à l'espace. Elle enrichit considérablement sa boîte à outils en proposant des notions nouvelles comme celles de "champs migratoires", d'“espaces migratoires", de "territoires de la mobilitét" ou encore de "système migratoire". La question des migrations et des mobilités est désormais un champ d'étude bien balisés et les programmes scolaires de géographie, longtemps marqués par l'approche classique des migrations, semblent depuis peu mieux prendre en compte cette bifurcation épistémologique.

\section{Les migrations, un}

objet secondaire dans

la géographie scolaire?

Le temps d'une géographie peu encline à s'intéresser au mouvement semble a priori révolu au regard des programmes scolaires contemporains. Au collège comme au lycée, les nouveaux programmes de 2009-2010 accordent une place importante aux questions migratoires : en classe de quatrième par exemple, $10 \%$ de l'année en géographie doivent être consacrés à l'étude des mobilités humaines ${ }^{6}$. Au lycée, alors que les programmes de géographie ont élargi leur approche des migrations à celle plus générale des mobilités, ceux d'histoire se sont particulièrement enrichis en matière d'étude des migrations et

1. Cet article est une version abrégée de l'intervention réalisée par l'auteur dans le cadre des “Deuxièmes rencontres universitaires et scolaires autour de l'enseignement de l'histoire-géographie", au lycée français Blaise-Pascal d’Abidjan en avril 2012. 2. Pierre George, Les Migrations internationales, Paris, PUF, 1976. 3. Gildas Simon, “'́espace des travailleurs tunisiens en France. Structures et fonctionnement d'un champ migratoire international”, thèse d'État de géographie, université de Poitiers, 1979. 4. Laurent Faret, Les Territoires de la mobilité. Migrations et communautés transnationales entre le Mexique et les États-Unis, Paris, CNRS éd., 2003. 5. Le Festival international de géographie de Saint-Dié des Vosges consacrait dès 1997 le thème de ses rencontres à “La planète 'nomade”, puis, en 2005, au “monde en réseaux", tandis qu'en 2010 "Les mobilités" devenaient une "question" à l'agrégation de géographie. 6. Bulletin officiel (B.O.) spécial n 6 du 28 août 2008. 
de l'immigration?. Mais, au-delà de leur place formelle dans les programmes, comment les phénomènes migratoires sont-ils traités ? Plus rarement étudiées pour ce qu'elles sont que pour ce qu'elles disent, les migrations sont en fait mobilisées pour révéler des problématiques d'ordre plus général. Analyser les flux migratoires de part et d'autre du Bassin méditerranéen est, par exemple, un moyen utile d'indiquer la fracture Nord-Sud, tandis que leur étude (volume, composition, destination) à l'échelle mondiale doit permettre de lire l'intégration des territoires à la mondialisation. Très souvent, l'étude des migrations s'arrête à cette première observation et, comme le déplore Gildas Simon, elles ne sont que rarement considérées comme un "phénomène social total" Il est également possible de noter que leur analyse prend pour support une série de repères spatiaux finalement assez limitée non seulement dans les programmes officiels, mais aussi dans les manuels. Certains espaces constituent ainsi de véritables lieux types des migrations avec toutes les limites de la stéréotypie que cela comporte (le Bassin méditerranéen et la frontière États-Unis/ Mexique).

\section{Des migrations "hors sol" ?}

Pour un enseignant, approcher la question des migrations passe inévitablement par l'étude des cartes de flux, notamment à l'échelle mondiale. Cette étape est nécessaire, mais la seule approche quantitative concourt à donner une image déshumanisée des migrations et des migrants. II n'est pas ici question de parcours individuels ou collectifs, mais d'un ensemble de flux matériels, dont l'élève peut difficilement imaginer à quelle réalité ils correspondent pour un individu. Pour paraphraser Armand Frémont évoquant le lien entre la société française et son territoire, les migrants "ne se comportent pas exactement comme des fourmis sur une plaque de verreg", mais comme des acteurs aux stratégies définies créant leur territoire de vie et prenant pleinement la mesure des rugosités physiques et politiques de l'espace qu'ils parcourent. Cette mise en flux des migrations interroge, enfin, la conception de l'espace sous-jacente. Conformément à l'approche fonctionnaliste de l'espace migratoire, l'analyse spatiale reste binaire (pays d'accueil versus pays de départ, immigration versus émigration), alors même que, d'une part, les circulations (retours provisoires, transferts d'argent, réseaux familiaux...) et les itinéraires des migrants sont de plus en plus complexes ${ }^{10}$ et que, d'autre part, la plupart des espaces migratoires sont devenus plurifonctionnels (espaces de départ, de transit, d'arrivée...). Avec cette approche, les mobilités peuvent rapidement être assimilées à des facteurs de perturbation du pavage territorial mondial". C'est aussi sans doute en raison de cette vision déshumanisée des migrations que la figure du migrant est régulièrement mobilisée dans les manuels.

\section{Le migrant, un "présent-absent"}

Parce que le choix de n'aborder les migrations que par les cartes occulte la réalité des parcours migratoires, le migrant peut rapidement être "absent en tant qu'individu' $u^{12}$. Mais, parce que la question

7. En seconde, le chapitre introductif d'histoire intitulé “Les Européens dans le peuplement de la Terre" permet une première approche des migrations internationales, alors qu'elle était auparavant réalisée en géographie. De la même manière, en première générale, l'étude approfondie de l'immigration en France, traitée auparavant en géographie dans le chapitre sur "La mobilité des hommes", est désormais réalisée en histoire. 8. Gildas Simon, La Planète migratoire dans la mondialisation, Paris, Armand Colin, 2008. 9. Armand Frémont, La France. Géographie d'une société, Paris, Flammarion, 1988. 10. Dans le cas des demandeurs d'asile, Olivier Clochard a montré ces "périples migratoires complexes et variés", comme le prouve le passage de migrants indiens ou sri-lankais à destination de l'Europe par le Mali, la Côte d'Ivoire ou l'Afrique du Sud. Voir Olivier Clochard, "Le jeu des frontières dans l'accès au statut de réfugié. Une géographie des politiques européennes d'asile et d'immigration”, thèse de géographie, université de Poitiers, cité par Gildas Simon, op. cit. 11. Denis Retaillé, “L'espace mobile”, in Benoit Antheaume, Frédéric Giraut (dir.), Le territoire est mort. Vive les territoires! Une (re)fabrication au nom du développement, Paris, IRD, 2005, pp. 175-202. 


\section{MÉMOIRE}

du fait migratoire est fréquemment présentée en terme d'immigration du seul point de vue du pays d'accueil, le migrant, qui par procédé métonymique généralisant est souvent le clandestin, peut aussi être présent de manière récurrente, voire obsessionnelle, dans l'enseignement des migrations. Comme le démontre l'historienne et inspectrice pédagogique régionale Marie Lavin, qui a étudié une vingtaine de manuels scolaires d'histoire, de géographie et d'éducation civique, les images de l'immigrant sont le

Pour un enseignant, approcher la question des migrations passe inévitablement par l'étude des cartes de flux, notamment

à l'échelle mondiale. Cette étape est nécessaire, maisla seule approche quantitative concourt à donner une image déshumanisée des migrations et des migrants. plus souvent "porteuses d'informations 'négatives " 13 ".

Trois grandes représentations du migrant ressortent. II est premièrement dépeint comme le responsable potentiel d'un ensemble de problèmes venant bouleverser un "état d'équilibre", à l'image de ce manuel de première L, ES, S (2011) qui note que "depuis 1973, l'Europe s'efforce d'endiguer le flot des clandestins affluant d'Afrique noire ou d'Asie". Aucune donnée chiffrée ne vient étayer l'affirmation, ce qui peut encourager chez l'élève une vision catastrophiste de l'immigration en Europe. À l'autre extrémité, c'est la vision de la victime permanente, le migrant est ainsi présenté comme un individu fuyant une situation de guerre et/ou de misère économique et sociale ; l'image joue alors un rôle déterminant tant par sa vertu didactique que par l'émotion qu'elle suscite chez l'élève ${ }^{14}$. Enfin, le migrant peut tout aussi bien être invisible et sa présence simplement suggérée. L'illustration est souvent la même : un mur ou une haute barrière métallique surmonté de fils barbelés et de miradors est surveillé par une patrouille de la Border Patrol ou de la Guardia Civil. Le migrant est absent de l'image mais son ombre plane de façon omniprésente : il est attendu, car traqués.

Bien qu'elles soient moins nombreuses, les manuels font également état d'images plus positives de la figure du migrant, particulièrement lorsqu'il s'agit d'évoquer la question de l'intégration aux États-Unis. Tous niveaux confondus, le parcours de Barack Obama, à la tête de la nation de migrants par excellence, reste un exemple récurrent dans les manuels et il est présenté comme le symbole de l'intégration à l'américaine ${ }^{16}$. Notons que dans le cas français, la question de l'intégration est traitée en histoire et, de ce fait, les manuels de géographie ne présentent pas, ou alors de manière très exceptionnelle, d'images positives du migrant. Atout ou problème potentiel, héros ou invisible, la présentation iconographique du migrant sort difficilement d'une lecture binaire. Dès lors, comme le suggère Gildas Simon ${ }^{17}$, l'approche globale du fait migratoire considérant les migrants comme des acteurs autonomes et légitimes, participants de plainpied à la mondialisation, permettrait de dépasser ces représentations extrêmes du migrant.

12. Paul Clerc, "Enseigner les migrations internationales : une demande de l'institution pour une nécessité civique”, conférence à l'IUFM d'Aix-Marseille, 19 novembre 2003. 13. Marie Lavin, “L'image des immigrés dans les manuels scolaires”, in Diversité, $n^{\circ}$ 149, 2007, pp. 97-103. 14. Des photographies d'immigrants africains entassés dans des embarcations sommaires tentant de traverser la Méditerranée, et généralement arraisonnés par les forces de l'ordre, illustrent de nombreux manuels et prolongent les images données par les médias 15. Ainsi, dans un manuel de première L, ES, S, cette image de la frontière espagnole à Melilla, constituée d'une barrière métallique surveillée au premier plan par une voiture de la Guardia Civil. Outre la photographie, la question à laquelle doit répondre l'élève ne mentionne aucunement le migrant: "Pourquoi l’Espagne a-t-elle édifié un dispositif frontalier à Melilla (enclave espagnole au Maroc) ?” Notons une ultime incarnation du migrant absent avec cette photographie d'une mosquée à Marseille, tirée d'un manuel de première (2011), qui incite l'élève à expliquer sa présence dans le tissu urbain par le fait migratoire. Le migrant est ainsi identifié comme musulman. 16. Un manuel de terminale fait un portrait de Barack Obama à la lumière de ses origines cosmopolites et immigrées : "Barak Hussein Obama est né le 4 août 1961 à Honolulu, Hawaii. Son père, économiste, était un Kenyan de l'ethnie Luo, alors que sa mère est une descendante de Jefferson Davis, le président des États confédérés d'Amérique, également d'ascendance cherokee. Barack Obama a également une ascendance irlandaise, un de ses grands-pères a émigré du comté irlandais d'Offaly." La note va jusqu'à signaler que "Barack'signifie 'béni' en hébreu, en arabe et en swahili". 17. Gildas Simon, "Penser globalement les migrations", in Projet, n² 272, 2002 [en ligne]. 


\section{Vers une approche globale des faits migratoires : migrations, territoires et identités}

Les nouveaux programmes de collège et de lycée mis en place à partir de la rentrée 2009 semblent avoir pris acte du tournant épistémologique dont les bases ont été posées dans les années 1970-1980. Au collège, la démarche imposée par exemple en quatrième pour l'étude de cas d'un flux migratoire du Maghreb vers l'Europe (thème 1) invite clairement à considérer les migrations de manière globale et surtout transnationale ${ }^{18}$. Tout en appréhendant l'étude de manière très concrète, puisque la fiche ressource précise qu'elle "peut être menée en suivant un itinéraire individuel", le professeur a la possibilité de proposer aux élèves une approche plus complexe des migrations en s'inspirant de certains concepts tels ceux de champ migratoire ou de circulation migratoire (sans d'ailleurs qu'il soit nécessaire de les nommer au collège). Ils permettent non seulement d'englober toutes sortes de flux (retours périodiques au pays, visite des membres de la famille dans les lieux d'installation, transferts financiers, autres liens affectifs, culturels), mais aussi de dépasser la conception figée du territoire. Sans aller jusqu'à confondre migration et circulation, car si "migrer est ou peut être une fin en soi, circuler ne l'est pas' 19 ", cette approche suppose que l'ordre sédentaire n'est pas seul à produire du territoire.

Il convient de réfléchir, non plus sur le sens donné à chaque lieu pris isolément, mais sur l'articulation des territoires entre eux. Conformément à leur rôle de laboratoire des programmes d'his- toire-géographie, les filières technologiques au lycée proposent des clés assez novatrices pour l'étude des migrations. Bien que facultatif, le sujet d'étude consacré à la diaspora chinoise en première STMG permet de repenser les questions migratoires. L'approche est, à plus d'un titre, originale : pour la première fois, ce chapitre conjugue regard historique et démarche géographique sans les distinguer. De plus, ce thème invite à réfléchir avec les élèves aux espaces de la diaspora et à leur imbrication à toutes les échelles géographiques (de sa diffusion mondiale à son inscription à l'échelle locale au sein de quartiers dans une ville), au lien entre puissance (politique, économique, culturelle) et migration, à la thématique de l'intégration ou encore à la dialectique complexe identité-migration. Sur ce dernier point, il peut être utile de montrer la diaspora comme une construction identitaire transnationale ${ }^{20}$ : parce que les lieux de référence de la population diasporique sont multiples, les identités qu'elle porte le sont également. Ce constat peut être généralisé aux migrants et aux enfants d'immigrés dont l'identité se construit et se recompose en fonction du degré de relation et de tensions qui se jouent entre le lieu de départ et le lieu d'arrivée, mais aussi en fonction de la charge symbolique qui y est investie ${ }^{21}$.

La migration, tout comme la mémoire et le passé migratoires, pose donc la question de la construction identitaire, qui ne peut pas se résumer à une identité exclusive et figée. En juxtaposant des territoires différents, les migrations participent de la production d'identités polymorphes, qui dépassent le seul cadre national. Néanmoins, les migrations naviguent entre contrainte et liberté, car le cadre politique garde encore toute sa pertinence. 


\section{MÉMOIRE}

\section{Pour une approche géopolitique des migrations}

Il semble nécessaire de saisir les migrations dans les cadres du pouvoir, car même si la mondialisation économique semble avoir affaibli le rôle des États-nations, le référent national et étatique reste essentiel en matière de politique migratoire et conditionne en cela les flux à l'échelle locale, continentale et mondiale. La démarche géopolitique telle que bâtie par Yves Lacoste ${ }^{22}$ offre une boîte à outils particulièrement efficace et opérante pour, par exemple, traiter certaines études de cas au lycée, comme celle de l'espace saharien (en terminale) ou de l'interface méditerranéenne (en première). Elle peut être menée en trois étapes fondamentales : la première consiste à partir des représentations que chacun des protagonistes a du territoire et des migrations. Même inexacte, une représentation géopolitique peut avoir une formidable capacité mobilisatrice et de grandes conséquences territoriales. Les discours de certains dirigeants européens brandissant le risque d'une amplification des flux migratoires servent souvent à justifier le renforcement des frontières européennes au sein de l'espace Schengen, et leur externalisation en assignant les États du sud de la Méditerranée (Maroc, Tunisie, Libye) au rôle de gardiens du glacis migratoire ${ }^{23}$. S'y ajoute la question de l'enjeu sécuritaire qui met l'accent sur la lutte contre le terrorisme : dans le contexte post11-Septembre, l'équation, voire l'amalgame, "clan- destins = terroristes" est un nouvel argument, qui permet là aussi de justifier un contrôle accru des migrations transfrontalières ${ }^{24}$. Dans un second temps, il convient de faire appel au raisonnement diachronique car l'accroissement des migrations à destination de l'Europe est en fait un phénomène relativement récent, qui date des années 1990 , alors que, parallèlement, les circulations transsahariennes s'inscrivent dans une profondeur historique, qui permet de remettre en cause la représentation courante d'un Sahara immobile ${ }^{25}$. Enfin, toute démarche géopolitique doit passer par une analyse diatopique, c'est-à-dire à différents niveaux d'analyse. Si à l'échelle locale (détroit de Gibraltar ou détroit de Sicile-Lampedusa), les flux migratoires en provenance d'Afrique subsaharienne et à destination de l'Europe paraissent conséquents, ils perdent de leur signification si on les rapporte à l'ensemble du Bassin méditerranéen : la voie méditerranéenne n'est plus la principale porte d'entrée des migrants vers l'Europe, les frontières migratoires s'étant déplacées vers l'est du continent. Par ailleurs, il convient de se situer à plus petite échelle pour s'apercevoir que de nombreux migrants subsahariens choisissent désormais de redéfinir leur trajectoire vers Israël et surtout vers les pays du Golfe via l'Égypte, où la forte croissance économique liée à l'exploitation du pétrole s'accompagne d'opportunités en matière d'emplois. Sans doute est-ce le résultat de la fermeture grandissante des frontières européennes, mais cela révèle aussi que les migrations sahariennes se mondialisent à leur tour. La démarche géopolitique permet de décentrer un regard bien

22. Voir le riche préambule d'Yves Lacoste dans Yves Lacoste (dir.), Dictionnaire de géopolitique, Paris, Flammarion, 1993 ; Géopolitique. La longue histoire d'aujourd'hui, Paris, Larousse, 2006, et la revue Hérodote. 23. Olivier Clochard, “Le contrôle des flux migratoires aux frontières de l'Union européenne s'oriente vers une disposition de plus en plus réticulaire”, in Carnets de géographes, $\mathrm{n}^{\circ}{ }^{1}, 2010$ [en ligne]. 24. Notons aussi que de l'autre côté de la Méditerranée, lorsque Kadhafi amplifiait les flux migratoires, il s'agissait pour ce dernier d'apparaître à la fin des années 1990 en position de force pour négocier la sortie d'embargo ou, en 2011, de menacer de ne plus jouer son rôle de dernier rempart après l'Europe en cas d'une intervention de l'Otan en Libye. Face à l'UE le pressant de durcir sa politique de surveillance des frontières et jouant sur le chantage au développement, le Maroc a également adopté une stratégie de victime des flux en provenance d'Afrique subsaharienne en diffusant des images spectaculaires de migrants à l'assaut des murs de Ceuta et de Melilla. Il a ainsi réussi à marchander sa politique d'endiguement migratoire en obtenant de substantielles aides économiques de la part de l'UE. Voir Guillaume Le Boëdec, "Le détroit de Gibraltar. Les limites d'un espace modèle de lutte européenne contre les migrations irrégulières”, in EchoGéo, n², 2007 [en ligne]. 25. Julien Brachet, Armelle Choplin, Olivier Pliez, "Le Sahara entre espace de circulation et frontière migratoire de l'Europe”, in Hérodote, n 142, 2011, pp. 163-182. 
souvent focalisé sur les seules problématiques européennes.

\section{L'immigration, sujet d'histoire... ou de géographie?}

Pour terminer, il peut être utile de s'interroger sur la place des questions d'immigration dans les programmes de géographie. Comme mentionné cidessus, l'étude de l'immigration en France était traitée en géographie (en première) avant la refonte des programmes en 2010. Elle est désormais réalisée en histoire ${ }^{26}$ : sans doute est-ce le résultat d'une juste réparation d'une question jusqu'alors ignorée dans l'enseignement de I'histoire, comme l'a démontré l'historien Benoît Falaize, chargé d'études et de recherche à l'INRP (Institut national de recherche pédagogique) ${ }^{27}$. Maintenant que I'histoire a repris "ses droits" sur les questions d'immigration, la géographie ne serait-elle plus légitime pour la traiter ? Cette question mérite d'être posée face au paradoxe suivant: le thème de l'immigration reste toujours un sujet majeur pour l'étude des États-Unis dans les programmes de géographie, alors qu'il est désormais traité en histoire dans le cas de la France. Plus fondamentalement, ce rééquilibrage révèle aussi la difficulté des programmes de géographie à intégrer les faits politiques et l'échelon national ${ }^{28}$ dans leur champ d'action. Pourtant, une géographie, voire une géopolitique de l'immigration ne serait-elle pas tout aussi justifiée et donc légitime ? L'entrée par les territoires, à travers, par exemple, l'étude des villes françaises en première, pourrait être tout à fait pertinente pour traiter cette question. Car l'étude des émeutes de 2005 ayant secoué les banlieues françaises doit-elle être réalisée en histoire, comme certains manuels de première le suggèrent, ou doit-elle être faite en géographie ? Des géographes comme Jérémy Robine $^{29}$ ont démontré toute l'utilité du raisonnement géographique et géopolitique pour comprendre les caractéristiques d'un ghetto (enclavement géographique, habitat dégradé, ségrégation sociale, fort taux d'habitants d'origine immigrée, conditions socio-économiques difficiles, etc.) et les rivalités territoriales qui y sont liées. Entre histoire immédiate et géopolitique, la question de l'immigration est bien un sujet qui nécessite à la fois les outils de l'historien et ceux du géographe. L'enseignement des phénomènes migratoires au collège comme au lycée s'est indéniablement enrichi à la suite du renouvellement des études géographiques sur ce thème depuis les trente dernières années. L'approche globale des migrations ouvre de nouvelles perspectives en classe de géographie : meilleure prise en compte de la clé territoriale, raisonnement multiscalaire, réflexion sur les enjeux identitaires liés aux migrations, etc. Mais force est de constater qu'elle est encore invisible aux yeux de certains professeurs. Car, contrairement à la discipline historique qui a profondément renouvelé l'enseignement du fait migratoire à la suite d'une prise de conscience générale résultant de rapports et d'enquêtes approfondies, la révolution géographique sur cette question reste silencieuse. Pourtant, en intégrant les apports de la recherche, la géographie scolaire a toute sa place pour penser le thème des migrations, qui - il ne faudrait pas l'oublier - demeure aussi et avant tout une question civique fondamentale.

26. Programme de première. Thème 1 : "Croissance économique, mondialisation et mutations des sociétés depuis le milieu du XIXe siècle", chapitre 2 "Mutations des sociétés": "Une étude : l'immigration et la société française au XXe siècle." 27. Notons que, dès 1986, l'historien Gérard Noiriel a montré dans un article que l'histoire de l'immigration était un champ minoré, voire occulté dans l'histoire nationale. Voir Gérard Noiriel, “L'immigration en France, une histoire en friche”, in Annales économies, sociétés, civilisations, vol. 41, n 4, 1986, pp. 751769 ; Benoît Falaize, Enseigner l'histoire de l'immigration à l'école, Paris, CNHI/INRP, 2008. 28. Yves Lacoste, La Géopolitique et le Géographe, Paris, Choiseul, 2010 ; Rémy Knafou, "Mais où est donc passée la France ?”, in Le Monde, 7 octobre 2011. 29. Jérémy Robine, Les Ghettos de la nation. Ségrégation, délinquance, identités, islam, Paris, Vendémiaire, 2011. 